\title{
Interruptions in medication administration: are we asking the right questions?
}

\author{
Anne Marie Rafferty, ${ }^{1,2}$ Bryony Dean Franklin ${ }^{2,3}$
}

${ }^{1}$ Florence Nightingale Faculty of Nursing and Midwifery, King's College London and PTSRC Imperial College London, London, UK

${ }^{2}$ NIHR Imperial Patient Safety Translational Research Centre London, UK

${ }^{3}$ Pharmacy Department, UCL School of Pharmacy and Imperial College Healthcare NHS Trust, London, UK

\section{Correspondence to}

Bryony Dean Franklin, Professor of Medication Safety, UCL School of Pharmacy and Imperial College Healthcare, London;

bryony.deanfranklin@imperial. nhs.uk

Received 17 April 2017 Accepted 17 April 2017 Published Online First 8 June 2017

CrossMark

To cite: Rafferty AM, Franklin BD. BMJ Qual Saf 2017;26:701-703.
The nature of today's healthcare practice makes interruptions, distractions and multitasking commonplace, even during complex and high-risk tasks. ${ }^{1-3}$ Interruptions are often cited as a problem in medication safety, particularly in relation to nurses administering medication. ${ }^{14}$ Previous studies ${ }^{56}$ suggest an association between interruptions and medication administration errors. While a direct causal relationship remains to be proven, reducing interruptions during medication administration to decrease multitasking and cognitive load represents a generally accepted goal. ${ }^{14}$

In this issue of BMJ Quality and Safety, Westbrook ${ }^{7}$ and colleagues report a cluster randomised controlled trial of a bundled intervention to reduce interruptions during medication administration in a hospital using paper-based prescribing. This well-designed feasibility study tested a bundled intervention based on 'Do Not Disturb' vests and the education of healthcare professionals, patients and visitors. At baseline, nurses experienced 57 interruptions per 100 dose administrations, with over 85\% unrelated to the dose of medication being prepared. The intervention moderately reduced these non-medication-related interruptions, with intervention nurses experiencing 15 fewer interruptions per 100 dose administrations compared with control nurses. However, the study also included a survey in which participating nurses characterised the vests as time consuming and uncomfortable. Only 48\% supported the intervention becoming hospital policy. Given the likely small impact of this modest reduction in interruptions on medication administration error rates, challenges to sustainability and the availability of alternate interventions, the authors conclude that wearing such vests does not represent best use of resources.

So where do we go from here? We suggest several points to consider. First, to find a way forward, as Westbrook et al point out, we may need to understand the causes and nature of interruptions in more detail. The present study separated interruptions that related to the specific dose of medication being prepared from all other types of interruption. This is a useful first step, and the results indicate a reduction in the latter 'non-medication-related' interruptions. However, the appropriateness or preventability of these interruptions was not considered further. Some, possibly many, may be completely unnecessary and avoidable. Others, however, even if not related to the dose of medication concerned, could be considered essential. For example, an interruption may be an urgent request for help if the patient in the next room is having a cardiac arrest or to inform the nurse that a dose of medication due to the next patient must not be given. Assuming that all interruptions that do not relate to the dose being given are inappropriate may, therefore, represent oversimplification of a complex situation. It is also possible that the causes and consequences of interruptions differ between paperbased and electronic medication administration systems.

Second, we may also need to consider the consequences of not interrupting. It is increasingly recognised that many interventions have unintended consequences as well as those intended. Interruptions are likely to be a natural by-product of an experienced nurse's role in supervising, communicating and coordinating the process of care. A nurse will be involved in a myriad of activities 
involving many contact points in the care continuum, within a complex web of relationships, during the course of each shift. The nurse is often the hub for these contacts and relationships, and so if at least some interruptions represent important communication, we need to understand what happens if this information is not communicated or is communicated differently-at a later time, to someone else or via an alternate route. The risk of interruption may also be linked to seniority if the senior person is a gatekeeper for other tasks such as the administration of controlled drugs, although no association between interruption rate and seniority was identified in another high-risk healthcare context. ${ }^{5}$ A requirement not to interrupt may also result in anxiety among patients and family members as well as other members of the healthcare team, particularly more junior staff, who will have to decide what might be important enough to justify an interruption to a nurse administering medication or what to do instead. To take this area forward, we therefore need to study the nuances of the relevance, importance and urgency of information currently communicated as interruptions, as well as the causes and consequences of both interrupting and not interrupting.

Taking into account these points, together with the findings of the study by Westbrook et al, ${ }^{7}$ we therefore need to consider whether other interventions may be more appropriate, and, if so, which. Interventions may relate to reducing the risk from interruptions or to other unrelated aspects of medication administration. In relation to reducing the impact of interruptions, as well as reducing the frequency of unnecessary and avoidable interruptions, we may need to consider how the recipients of interruptions can mitigate their impact or how best to use other ways of communicating important information. Staff may benefit from training in non-technical skills such as situation awareness, task management and coping with stress, ${ }^{8}$ more concrete guidance around what is acceptable in terms of help-seeking behaviours during medication rounds and a calm functional working environment. We rarely train or prepare practitioners to cope with the chaos and pace of workflow or the constant switching between different types of task and different work modesemotional, technical, physical, cognitive and intellectual. It may also be that interrupting staff administering medication represents an overly relaxed attitude to medication and other tasks that require concentration, and so wider culture change may be needed, above and beyond specific interventions. Another approach may be to clearly identify a member of staff who can be interrupted, drawing on various approaches to quality improvement with healthcare that advocate standardisation and streamlined division of labour. ${ }^{9}$ Alternatively, as Westbrook and colleagues suggest, resources may be better used on other interventions such as introducing computerised prescribing and electronic medication administration record systems where these are not yet in place.
But perhaps we are approaching the problem from the wrong end of the telescope. We are told that nurses did not like wearing the vests, finding them uncomfortable and difficult to take on and off, which raises the question of the extent to which they were involved in selecting the interventions or designing the vests. Co-designing interventions with those involved is increasingly being used as an approach to quality improvement and service redesign. ${ }^{10} 11$ Perhaps the problem needs reframing first with nurses, patients other healthcare professionals and visitors as well as with patient safety experts, to identify the most appropriate solutions.

In conclusion, the work of Westbrook et al is helpful in raising these issues; we now need to reframe the questions in order to decide if and how we should address interruptions and distractions in medication administration, or whether we should be looking elsewhere for the solutions.

Funding The authors are partially funded by the National Institute for Health Research (NIHR) Imperial Patient Safety Translational Research Centre.

Disclaimer The views expressed are those of the authors and not necessarily those of the National Health Service, the NIHR or the Department of Health. The author BDF is an editor at BMJ Quality and Safety.

\section{Competing interests None declared.}

Provenance and peer review Commissioned; internally peer reviewed.

(C) Article author(s) (or their employer(s) unless otherwise stated in the text of the article) 2017. All rights reserved. No commercial use is permitted unless otherwise expressly granted.

\section{REFERENCES}

1 Bower R, Jackson C, Manning JC. Interruptions and medication administration in critical care. Nurs Crit Care 2015;20:183-95.

2 Larcos G, Prgomet M, Georgiou A, et al. A work observation study of nuclear medicine technologists: interruptions, resilience and implications for patient safety. BMJ Qual Saf 2016:bmjqs-2016-005846.

3 Weigl M, Müller A, Vincent C, et al. The association of workflow interruptions and hospital doctors' workload: a prospective observational study. BMJ Qual Saf 2012;21:399-407.

4 Raban MZ, Westbrook JI. Are interventions to reduce interruptions and errors during medication administration effective? A systematic review. BMJ Qual Saf 2014;23:414-21.

5 Westbrook JI, Woods A, Rob MI, et al. Association of interruptions with an increased risk and severity of medication administration errors. Arch Intern Med 2010;170:683-90.

6 Blignaut AJ, Coetzee SK, Klopper HC, et al. Medication administration errors and related deviations from safe practice: an observational study. J Clin Nurs 2017.

7 Westbrook JI, Li L, Hooper TD, et al. Effectiveness of a 'Do not interrupt' bundled intervention to reduce interruptions during medication administration: a cluster 
randomised controlled feasibility study. BMJ Qual Saf 2017:bmjqs-2016-006123.

8 Sevdalis N. Non-technical skills and the future of teamwork in healthcare settings. London: The Health Foundation, 2013. http://patientsafety.health.org.uk/sites/default/files/resources/ non_technical_skills_and_the_future_of_teamwork_in healthcare_settings.pdf. (accessed 8 Apr 2017).
9 Critchley S. Improving medication administration safety in a community hospital setting using Lean methodology. J Nurs Care Qual 2015;30:345-51.

10 Robert G, Cornwell J, Locock L, et al. Patients and staff as codesigners of healthcare services. BMJ 2015;350:g7714.

11 Batalden M, Batalden P, Margolis P, et al. Coproduction of healthcare service. BMJ Qual Saf 2016;25:509-17. 